\title{
Estudio transversal sobre el dolor y funcionalidad del pie en pacientes diabéticos no neuropáticos y pacientes no diabéticos
}

\author{
Cross-sectional study on the pain and functionality of the foot in non-neuropathic diabetic \\ patients and non-diabetics patients
}

José María Domínguez-Olmedo y María Reina-Bueno

Departamento de Podología, Universidad de Sevilla. Sevilla, España

Palabras clave:

Diabetes mellitus, pie diabético, dolor, cuestionario de salud del paciente, podología rendimiento funcional, complicaciones de la diabetes, pie.

\section{Resumen}

Objetivo: El objetivo principal de este trabajo fue comprobar si el dolor y la funcionalidad del pie eran diferentes en pacientes con y sin diabetes mellitus.

Pacientes y métodos: La muestra fue obtenida en el Área Clínica de Podología de la Universidad de Sevilla, y estuvo compuesta por 101 pacientes divididos en dos grupos: 57 sujetos con diabetes mellitus y 44 personas sin diabetes mellitus. Se emplearon los cuestionarios Foot Function Index y el Cuestionario de Manchester sobre el dolor y la discapacidad del pie para medir el dolor y la funcionalidad del pie.

Resultados: Existen diferencias significativas entre los dos grupos en el componente funcional del Cuestionario de Manchester sobre el dolor y la discapacidad del pie, donde la mejor puntuación la obtiene el grupo sin diabetes mellitus. Para el resto de valores no existen diferencias significativas.

Conclusiones: El grupo de pacientes con diabetes mellitus presentó mayor limitación funcional de acuerdo con los resultados obtenidos del Cuestionario de Manchester sobre el dolor y la discapacidad del pie. El dolor fue el mismo en presencia de diabetes mellitus o no.

\section{Abstract}

Objective: The main objective of this work was to verify if the pain and functionality of the foot were different in patients with and without diabetes mellitus.

Method: The sample was obtained in the podiatric clinic area of the University of Seville, and consisted of 101 patients divided into two groups: 57 patients with diabetes mellitus and 44 persons without diabetes mellitus. The Foot function index and the Manchester foot pain and disability index were used to measure foot pain and functionality.

Results: There are significant differences between the 2 groups in the functional component of the Manchester foot pain and disability index, where the best score is obtained by group B. For the rest of the values there were no significant differences.

Conclusions: The group of patients with diabetes mellitus presented greater functional limitation according to the results obtained from the Manchester foot pain and disability index. The pain was the same in the presence of diabetes mellitus or not. 


\section{INTRODUCCIÓN}

Con el paso del tiempo la esperanza de vida de la sociedad ha ido aumentando, debido principalmente a los avances en las ciencias de la salud. Esto, unido a los malos hábitos de vida, ocasiona la aparición de enfermedades crónicas; entre las más prevalentes se encuentra la diabetes mellitus (DM) ${ }^{1}$.

El propósito de este trabajo fue comprobar si el dolor y la funcionalidad del pie eran diferentes en pacientes con y sin DM. No se han encontrado estudios en los que se investigue la funcionalidad y el dolor en la DM empleando los cuestionarios Foot Function Index (FFI) y el Cuestionario de Manchester sobre el dolor y la discapacidad del pie (MFPDI). A continuación se presentan los trabajos realizados en los últimos cinco años sobre este tema.

En 2015, Yang y cols. ${ }^{2}$ investigaron los cambios en las presiones plantares de los individuos con DM. En el estudio incluyeron 649 pacientes con DM y 808 participantes sin DM. El tiempo de contacto plantar y la fuerza máxima de presión eran mayores en el grupo con DM. Las diferencias significativas de presión y tiempo de contacto plantar pueden referirse a los factores de riesgo de desarrollar úlceras en el pie. En ese mismo año, Lamola y cols. ${ }^{3}$ estudiaron a 30 pacientes con DM. Los participantes presentaban dos modificaciones biomecánicas: reducción de la flexo-extensión del pie y la ampliación de la superficie de apoyo. Estos factores dan lugar a peor movilidad en el pie y peor control del equilibrio conforme aumenta la enfermedad. En 2016, Hsu y cols. ${ }^{4}$ observaron que los pacientes con DM presentan alto riesgo de caída. Realizaron un estudio en 14 pacientes con DM y 14 sin DM. Los pacientes con DM presentaban mayor debilidad muscular en la flexión de rodilla y en la aducción de cadera, aumentando así la demanda de los plantarflexores del pie, por lo que estos pacientes presentan mayor dificultad de evitar obstáculos, y por lo tanto mayor probabilidad de sufrir caídas. Finalmente, en 2019 , Hazari y cols. ${ }^{5}$, tras estudiar a 120 pacientes con DM y neuropatía periférica, concluyeron que estas personas presentaban cambios en sus parámetros biomecánicos, haciendo que aumente el riesgo de ulceración.

\section{PACIENTES Y MÉTODOS}

\section{Población de estudio}

Se ha realizado un estudio transversal, observacional, descriptivo e inferencial. Se realizó la comparación de dos grupos, uno de sujetos con DM (Grupo A) y un grupo control de pacientes sin estos problemas (Grupo B) $)^{6}$.

La muestra estuvo formada por 101 sujetos, 51 hombres (50.5\%) y 50 mujeres (49.5\%), divididos en dos grupos. El grupo A lo componían 57 pacientes con DM con una edad media de $62.46 \pm 14.93$ años y un IMC de media de $29.23 \pm 4.35$. El grupo B incluía a 44 participantes sin DM con una edad media de $64.73 \pm 15.68$ años y un IMC de media de $27.77 \pm 4.21$
En cuanto al tamaño muestral, el grupo A y B debía estar compuestos como mínimo por 42 pacientes cada uno, para un error tipo I de 0.05 , una potencia del $84 \%$ y con características similares en cuanto a género, edad e $\mathrm{IMC}^{6-8}$.

El grupo A presentaba una evolución de DM entre los 1 y los 46 años (15.61 \pm 10.29 años) y un $38.59 \%$ de los sujetos no conocían sus niveles de hemoglobina glicosilada.

El trabajo de campo se llevó a cabo en el Área Clínica de Podología de la Universidad de Sevilla. La recogida de datos se realizó desde enero de 2017 a julio de 2018. Este estudio fue aprobado por el Comité de Bioética de los Hospitales Universitarios Virgen Macarena y Virgen del Rocío de Sevilla, con código de referencia 0227-N-17.

\section{Criterios de inclusión y exclusión}

Para el grupo A, los criterios de inclusión fueron tener diagnóstico previo de DM y ser mayores de 18 años $^{9,10}$. Para el grupo B, los criterios de inclusión fueron ser mayores de 18 años y no tener diagnóstico de $\mathrm{DM}^{8}$. El criterio de diagnóstico de DM se basó en los establecidos por la Asociación de Diabetes Americana ${ }^{11}$.

Los criterios de exclusión fueron presencia de ulceración abierta y/o amputación previa del pie o miembro inferior ${ }^{12}$, neoplasia, debido a la necesidad de que los pacientes debían responder cuestionarios también se añadieron como criterios la demencia ${ }^{13,14}$, la dificultad de expresión y de movilidad ${ }^{9,10}$, y, tras la realización de una exploración neurológica básica, se excluyó a los pacientes que presentasen el menor síntoma de neuropatía periférica ${ }^{15}$.

\section{Variables de estudio}

Los cuestionarios fueron administrados por el investigador principal; eran dados a los pacientes y estos los rellenaban; las dudas eran aclaradas por el mismo investigador; tras completarlos, este los tomaba y almacenaba para su posterior análisis estadístico. Los cuestionarios son los siguientes.

El Cuestionario de Manchester sobre el dolor y la discapacidad del pie (MFPDI), usado para medir el dolor y la funcionalidad. Consta de 19 ítems, los 10 primeros tienen relación con la limitación funcional, los 5 siguientes se corresponden con la intensidad del dolor (aplicables en pacientes con neuropatía), los dos siguientes se refieren a la apariencia personal y los dos últimos se relacionan con la actividad y el trabajo del paciente. El valor total del cuestionario oscila entre 0 y 38 , siendo este último el peor estado de discapacidad posible ${ }^{16}$.

El Foot function index (FFI) es un cuestionario desarrollado para medir el impacto de las patologías del pie en la funcionalidad, distribuido en tres subescalas: discapacidad, limitación de la actividad y dolor. Los resultados son expresados en porcentajes, siendo $100 \%$ el peor estado funcional posible ${ }^{17}$.

También para la medición del dolor se empleó la escala numérica del dolor de la OMS; se trata de una línea de dividida en 10 partes iguales donde el paciente establece el nivel del 
dolor que padece; podía ser sin dolor (0), leve (1-3), moderado (4-7 y severo $(8-10)^{18}$.

\section{Análisis estadístico}

Para el análisis estadístico se utilizó el paquete estadístico IBM SPSS Statistics 22 para Windows. Las variables cualitativas se expresaron mediante tablas de frecuencia y las cuantitativas mediante medidas de centralización y dispersión: media, mediana, desviación estándar y valores máximos y mínimos.

Las pruebas de normalidad de las distribuciones de variables cuantitativas, en los grupos que componen la muestra, se realizó el test de Kolmogorov-Smirnov en el grupo A por tener un tamaño muestral superior a 50 (57); en el grupo B la normalidad se comprobó mediante el test de Shapiro-Wilk por tener un tamaño muestral inferior a 50 (44). En el grupo de DM la distribución normal la seguían el IMC, la hemoglobina glicosilada y el total del MFPDI.

Para determinar si existían diferencias significativas para los valores de las distintas variables, entre los grupos se utilizaron las siguientes pruebas estadísticas: para variables cuantitativas normales se realizó la prueba t de Student para muestras independientes; para variables cuantitativas no normales se utilizó la prueba U de Mann-Whitney; para variables cualitativas se utilizó el test de Chi cuadrado.

Para este tipo de análisis se ha tenido en cuenta un nivel de confianza del $95 \%$. Se considera que existe diferencias estadísticamente significativas si $p<0.05$.

\section{RESULTADOS}

Las Tablas I y II muestran los datos descriptivos de los cuestionarios FFI y MFDPI, respectivamente, en los tres grupos de participantes.

Entre los dos grupos no se encontraron diferencias significativas en los valores obtenidos en el FFI. Se encontraron diferencias significativas en relación con los valores obtenidos del componente funcional del MFPDI $(p=0.007)$. La puntuación más alta pertenece al grupo A, seguido por el grupo B. Para el resto de variables no existen diferencias significativas.

\section{DISCUSIÓN}

El propósito de este trabajo fue comprobar si el dolor y la funcionalidad del pie eran diferentes en pacientes con y sin

Tabla I. Descripción del FFI en la muestra.

\begin{tabular}{lllll}
\multirow{2}{*}{ FFI } & & Media & Desviación estándar & P-Valor \\
\hline \multirow{3}{*}{ Grupo A } & Dolor & 25.68 & 26.71 & 0.428 \\
\cline { 2 - 4 } & Discapacidad & 29.48 & 28.52 & 0.115 \\
\cline { 2 - 4 } & Limitación funcional & 7.64 & 14.83 & 0.508 \\
\cline { 2 - 4 } & Total & 22.97 & 21.71 & 0.148 \\
\hline \multirow{3}{*}{ Grupo B } & Dolor & 21.20 & 25.04 & 0.428 \\
\cline { 2 - 4 } & Discapacidad & 23.05 & 26.50 & 0.115 \\
\cline { 2 - 4 } & Limitación funcional & 5.00 & 10.74 & 0.508 \\
\cline { 2 - 4 } & Total & 18.42 & 19.64 & 0.148 \\
\hline
\end{tabular}

Tabla II. Descripción de cuestionario de MFPDI en la muestra.

\begin{tabular}{lllll} 
Cuestionario de Manchester & Media & Desviación estándar & P-Valor \\
\hline \multirow{4}{*}{ Grupo A } & Funcional & 7.12 & 5.75 & 0.007 \\
\cline { 2 - 5 } & Apariencia personal & 0.35 & 0.83 & 0.522 \\
\cline { 2 - 4 } & Dolor & 3.14 & 2.90 & 0.536 \\
\cline { 2 - 5 } & Trabajo & 1.18 & 1.59 & 0.074 \\
\cline { 2 - 5 } & Total & 11.79 & 9.37 & 0.052 \\
\hline \multirow{3}{*}{ Grupo B } & Funcional & 4.75 & 5.38 & 0.007 \\
\cline { 2 - 4 } & Apariencia personal & 0.50 & 1.15 & 0.522 \\
\cline { 2 - 4 } & Dolor & 2.73 & 3.16 & 0.536 \\
\cline { 2 - 4 } & Trabajo & 0.61 & 1.22 & 0.074 \\
\cline { 2 - 4 } & Total & 8.59 & 9.47 & 0.052 \\
\hline
\end{tabular}


DM. Se encontraron diferencias significativas en cuanto a la funcionalidad del MFPDI, en el que el grupo de pacientes con DM obtuvo peor puntuación. En el cuestionario FFI no se han encontrado diferencias estadísticamente significativas entre ambos grupos.

Estos cuestionarios han sido validados en múltiples países. En España, el FFI fue validado en 2013 por Paez-Moguer, Budiman-Mak y Cuesta-Vargas. Concluyeron que el FFI era un cuestionario útil a la hora de cuantificar la funcionalidad del pie en diferentes patologías ${ }^{19}$. El MFPDI fue validado al español por Gijón-Nogueron y cols. en 2014. Se llegó a la conclusión que esta herramienta era muy útil a la hora de medir el dolor frente a otros instrumentos ${ }^{16}$.

En la mayoría de los estudios sobre el FFI se habla de la gran utilidad de este instrumento en múltiples patologías dolorosas del pie, como reflejan Budiman-Mak y cols. que, en 2013, consideraron este cuestionario como pionero en la medición de la salud del pie, válido, reproducible y con una sensibilidad muy alta en distintas patologías limitantes del pie ${ }^{20}$. El FFI ha sido comparado incluso con el propio MFPDI por van der Zwaard y cols. en 2014, quienes establecieron una correlación moderada entre el MFPDI y el SF-12. Además determinaron que el FFI era el mejor instrumento para estudios transversales y que el MFPDI sería conveniente emplearlo en estudios longitudinales ${ }^{21}$. Es cierto que ha sido empleado en gran cantidad de estudios y que cada vez está siendo más utilizado ${ }^{16}$, por lo que se ha considerado conveniente incluirlos en este trabajo.

A continuación se procede a realizar la discusión con los trabajos presentados en los últimos 5 años sobre este tema.

De acuerdo con los resultados de Yang y cols. ${ }^{2}$, las presiones en el pie son mayores en el grupo DM que en el grupo control. Esto podría coincidir con el presente trabajo en que el componente funcional del MFPDI esté más afectado en el grupo con DM, ya que el peor reparto de presiones podría estar relacionado con una funcionalidad más pobre por parte del paciente. Al igual que en el estudio presentado por Lamola y cols. ${ }^{3}$, en el que establece que el paciente tendrá peor movilidad conforme va empeorando la enfermedad.

Los estudios de Hsu y cols. ${ }^{4}$ y Hazari y cols. ${ }^{5}$ coinciden también con los autores anteriores en que los pacientes con DM presentan diferencias biomecánicas en relación con los pacientes sin DM. Hsu y cols. ${ }^{4}$ Ilegan a destacar que el paciente con DM es más propenso a sufrir caídas y de esa manera poseer una capacidad funcional peor.

El presente trabajo coincide con los estudios mencionados en cuanto a la funcionalidad del pie, de acuerdo con los resultados obtenidos del componente funcional del MFPDI, el grupo más afectado es el de DM, por lo que la presencia de esta enfermedad hace más difícil la realización de actividades y tareas.

Gracias a los hallazgos que se han obtenido, se podría mejorar la adherencia al tratamiento por parte del paciente con DM mediante la creación de nuevas técnicas que ayuden a mejorar las limitaciones funcionales presentes en la enfermedad.
Este estudio presenta ciertas limitaciones que hacen que sus resultados deban ser interpretados con cautela. Cabe destacar que un gran porcentaje de pacientes no sabían el valor de la hemoglobina glicosilada; por lo tanto, no se puede determinar si la DM está controlada. Debido a este hecho, no se han podido relacionar las complicaciones y sintomatología con el control de la DM. No se tuvo en cuenta la presencia de factores externos a la DM, como puede ser la edad o el nivel educativo, entre otras opciones; se podrían haber conseguido resultados interesantes al respecto. La metodología cuantitativa en sí puede ser una limitación, puesto que usar cuestionarios cerrados hace que algunos matices importantes se pierdan. El uso de la metodología cualitativa podría ser interesante en un tema tan subjetivo como lo es la calidad de vida. No se tuvo en cuenta la presencia de enfermedades que produjesen limitación funcional, algo que podría haber hecho que el estudio tuviese una aplicación más específica y que será tenido en cuenta para futuros trabajos.

En conclusión, la DM produjo en los participantes de este trabajo limitación funcional de acuerdo con los resultados obtenidos del MFPDI. Tanto el FFI y el MFPDI son considerados cuestionarios válidos para la medición de problemas en el pie de pacientes con DM por su reproductibilidad y resultados que presentan. Si estos resultados son tenidos en cuenta, se podría decir que la limitación de la función es importante en la DM y el tratamiento debe estar orientado hacia este problema, de esta manera se podría mejorar la adherencia al tratamiento por parte del paciente de forma más efectiva. Cabe destacar la importancia de la actuación de un equipo multidisciplinar en el que el podólogo tendría un papel importante, orientando su actuación en la recuperación funcional, junto con el fisioterapeuta y el endocrinólogo para controlar la DM.

\section{CONFLICTO DE INTERESES}

Los autores declaran no tener ningún conflicto de intereses.

\section{FINANCIACIÓN}

Ninguna.

\section{BIBLIOGRAFÍA}

1. González-Consuegra RV, Verdú J. Calidad de vida relacionada con heridas crónicas. Gerokomos. 2010;21(3):131-9.

2. Yang C, Xiao H, Wang C, Mai L, Liu D, Qi Y et al. Variation of plantar pressure in Chinese diabetes mellitus. Wound Rep Reg. 2015;23(6):932-8. DOI: $10.1111 /$ wrr. 12331.

3. Lamola G, Venturi M, Martelli D, lacopi E, Fanciullacci C, Coppelli A, et al. Quantitative assessment of early biomechanical modifications in diabetic foot patients : the role of foot kinematics and step width. J Neuroeng Rehabil. 2015;12:98. DOI: 10.1186/s12984-015-0093-6.

4. Hsu W, Liu M, Lu T. Biomechanical risk factors for tripping during obstacle - Crossing with the trailing limb in patients with type II diabetes mellitus. Gait Posture. 2016;45:103-9. DOI: 10.1016/j. gaitpost.2016.01.010. 
5. Study AH, Hazari A, Maiya AG, Shivashankara KN. Foot Kinetic and Kinematic Profile in Type 2 Diabetes Mellitus with Peripheral Neuropathy. J Am Podiatr Med Assoc. 2019;109(1):36-49. DOI: 10.7547/17-059.

6. Argimon JM, Jiménez J. Métodos de investigación clínica y epidemiológica. $4^{\mathrm{a}}$ ed. Barcelona: Elsevier; 2013.

7. Ritchey FJ. Estadística para ciencias sociales. Potencial de la imaginación estadística. Madrid: McGraw-Hill; 2008.

8. Anderson D, Sweeney D, Williams T. Estadística para la administración y economía. Madrid: Learning C; 2008.

9. Oka RK, Sanders MG. The impact of type 2 diabetes and peripheral arterial disease on quality of life. J Vasc Nurs. 2005;23(2):61-6. DOI: 10.1016/j.jvn.2005.03.032.

10. Amer MS, Alsadany MA, Tolba MF, Omar OH. Quality of life in elderly diabetic patients with peripheral arterial disease. Geriatr Gerontol Int. 2013;13(2):443-50. DOI: 10.1111/j.1447-0594.2012.00928.x.

11. American Diabetes Association. Classification and Diagnosis of Diabetes: Standars of Medical Care in Diabetes-2018. Diabetes Care. 2018;41(suppl 1):S13-S27. DOI: 10.2337/dc18-S002.

12. Viadé J. Pie Diabético. Guía para la práctica clínica. Barcelona: Panamericana; 2013.

13. OMS. Cancer [Intenet]. OMS, 2020; consultado el 24 de octubre de 2018. Disponible en: http://www.who.int/es/news-room/fact-sheets/ detail/cancer

14. OMS. Demencia [Intenet]. OMS, 2020; consultado el 24 de octubre de 2018. Disponible en: http://www.who.int/es/news-room/fact-sheets/ detail/dementia
15. Keri KC, Samji NS, Blumenthal S. Diabetic nephropathy: newer therapeutic perspectives. J Community Hosp Intern Med Perspect. 2018;8(4):200-7. DOI: 10.1080/20009666.2018.1500423.

16. Gijon-Nogueron G, Ndosi M, Luque-Suarez A, Alcacer-Pitarch B, Munuera PV, Garrow A, et al. Cross-cultural adaptation and validation of the Manchester Foot Pain and Disability Index into Spanish. Qual Life Res. 2014;23(2):571-9. DOI: 10.1007/s11136-013-0507-5.

17. Martinez BR, Staboli IM, Kamonseki DH, Budiman-Mak E, Yi LC. Validity and reliability of the Foot Function Index (FFI) questionnaire Brazilian-Portuguese version. Springerplus. 2016;5(1):1810. DOI: 10.1186/ s40064-016-3507-4.

18. Ribeiro SBF, Carlos J, Pinto P, Ribeiro JB, Felix MMS, Barroso SM, et al. Dolor en las Unidades de Ingreso de un Hospital Universitario. Rev Bras Anestesiol. 2012;62(5):605-11. DOI: 10.1590/S003470942012000500001

19. Paez-Moguer J, Budiman-Mak E, Cuesta-Vargas Al. Cross-cultural adaptation and validation of the Foot Function Index to Spanish. Foot Ankle Surg. 2014;20(1):34-9. DOI: 10.1016/j.fas.2013.09.005.

20. Muradin I, van der Heide HJL. The foot function index is more sensitive to change than the Leeds Foot Impact Scale for evaluating rheumatoid arthritis patients after forefoot or hindfoot reconstruction. Int Orthop. 2016;40(4):745-9. DOI: 10.1007/s00264-016-3113-7.

21. van der Zwaard BC, Terwee CB, Roddy E, Terluin B, van der Horst HE, Elders PJ. Evaluation of the measurement properties of the Manchester foot pain and disability index. BMC Musculoskelet Disord. 2014;15:276. DOI: $10.1186 / 1471-2474-15-276$. 